\title{
CONF- $9606195-2$ \\ NONEQUILIBRIUM MOLECULAR DYNAMIC SIMULATIONS ON MASSIVELY PARALLEL SUPERCOMPUTERS
}

\author{
Ravi Bhupathiraju and Peter T. Cummings \\ Department of Chemical Engineering \\ University of Tennessee \\ Knoxville, TN 37996-2200
}

\author{
H. D. Cochran \\ Oak Ridge, TN 37831-6224 \\ Presented and published: \\ June 19-22, 1996 \\ Submitted to: \\ J. Comp. \& Math \\ June, 1996
}

Chemical Technology Division

Oak Ridge National Laboratory*

Intel Supercomputer User Group

(ISUG '96), Knoxville, Tennessee

RECEIVED

MAY 171996

Q. Sat:

The submitted manuscript has been authored

by a contractor of the U.S. Government under contract No. DE-AC05-960R22464. Accordingly, the U.S. Government retains a nonexclusive, royalty-free license to publish or reproduce the published form

of this contribution, or allow others to do so, for U.S. Government purposes.

*Managed by Lockheed Martin Energy Research Corp. for the U.S. Department of Energy under contract number DE-AC05-96OR22464. 


\title{
Nonequilibrium Molecular Dynamic Simulations on Massively Parallel Supercomputers
}

\author{
Ravi Bhupathiraju, Peter T. Cummings, and Hank D. Cochran \\ Department of Chemical Engineering \\ University of Tennessee, Knoxville, TN 37996 \\ and \\ Chemical Technology Division \\ Oak Ridge National Laboratory, Oak Ridge, TN 37831
}

\begin{abstract}
Large scale simulations of planar Couette flow in fluids using domain decomposition often require complex and/or expensive routines to handle the Lees-Edwards periodic boundary conditions. In this paper we present an efficient algorithm that reduces both complexity and the computational costs for such simulations. Shear viscosity results for the Weeks-Chandler-Anderson fluid obtained from large scale simulations using the new algorithm are also presented.
\end{abstract}

KEYWORDS: nonequilibrium molecular dynamics, deforming cell, Lees-Edwards, domain decomposition

\section{Introduction}

The new generation of distributed memory parallel supercomputers has provided a highly scalable environment for very large scale molecular simulations. Domain decomposition algorithms based on the link cell technique have been shown to take advantage of this virtually unlimited power, especially for molecular dynamics (MD) simulations of particles with short range interactions [1]. However, the efficiency of the parallel nonequilibrium molecular dynamics (NEMD) algorithms is restricted by the special periodic boundary conditions required for such cases. Simulations of planar Couette flow, for instance, apply Lees-Edwards periodic boundary conditions in order to avoid surface effects. Because of the sliding nature of the image cells above and below the central simulation cell under these periodic boundary conditions, complex communication patterns are required for bookkeeping of image particles on the processors at the shear boundaries and overhead costs are high [2]. Recently, a deforming cell method was proposed by Hansen and Evans 
[3] where the simulation cell is allowed to deform with the shear flow such that the shearing boundaries always align with the image cells. Whenever the simulation cell deforms by $45^{\circ}$ it is reset to prevent it from deforming indefinitely in one direction. The deforming cell method allows the parallel NEMD simulations use the same message passing as the parallel equilibrium molecular dynamics case. However, a larger link cell size is required for force calculations in this method so that particles in a link cell interact only with the particles in the same link cell or its immediate neighbors even at the maximum angle of deformation. The increase in link cell size substantially increases the computational time because nearly $180 \%$ more particles pairs must be considered in the force calculations in this case as compared to a rigid simulation cell case.

In this study, we present a modified deforming cell algorithm that allows us to perform NEMD simulations with almost negligible penalties due to the boundary conditions. The improvement is achieved by resetting the simulation cell at a shorter deforming angle thereby requiring a smaller link cell size. Performance tests on the Intel Paragon XP/S 150 showed that the overhead costs for the new method are less than $10 \%$ for the WeeksChandler-Andersen (WCA) fluid system. We also present low shear rate viscosity results for the WCA fluid system obtained by conducting large scale simulation experiments using the new algorithm.

\section{Algorithm}

The equations of motion for an N-body atomic fluid under planar Couette flow can be described by the isokinetic SLLOD dynamics:

$$
\begin{gathered}
\dot{\mathrm{r}}_{i}=\frac{\mathrm{p}_{i}}{m_{i}}+\mathrm{i} \gamma y_{i} \\
\dot{\mathrm{p}}_{i}=\mathbf{F}_{i}-\mathrm{i} \gamma p_{y i}-\alpha \mathbf{p}_{i} \\
\alpha=\frac{\sum\left(\mathrm{F}_{i} \cdot \mathrm{p}_{i}-\gamma p_{x_{i}} p_{y_{i} i}\right)}{\sum p_{i}^{2}}
\end{gathered}
$$

where $\mathbf{r}_{i}, \mathbf{p}_{i}$ are the position and peculiar momentum of particle $i, \mathbf{F}_{i}$ is the total force on it, $m$ is the mass, is the shear rate. The Gaussian thermostat multiplier $\alpha$ is introduced to remove the viscous heat generated irreversibly by the shear. The equations of motion are integrated using the 4th order Gear predictor corrector method.

The SLLOD equations of motion are implemented with Lees-Edwards periodic boundary conditions to simulate an infinite system under shear. Under these boundary conditions the infinite periodic images of the simulation box are translated in the shear direction depending on their instantaneous lattice vectors with respect to the simulation box and the strain rate gamma. Hence the particles moving out of the shearing faces of the simulation cell are translated by a distance $y \gamma t$ before being reintroduced in the opposite shearing face. The sign of the value depends on the direction in which the particle is moving out i.e. -ve for particles moving out in the positive y direction and 
+ve for the particles moving out in the negative $y$ direction). For large scale simulations using domain decomposition, this would result in complex and expensive message passing since the particle transfer pattern from one processor domain to another across the shearing boundaries changes continuously with time [2]. Using the deforming cell boundary conditions and allowing the individual processor domains to deform the shear flow, this problem reduces to the case of parallel equilibrium molecular dynamics. However, the overhead in terms of number of interactions considered during force calculation due to the increase in link cell size for the deforming case can be shown to be proportional to $1 / \cos ^{3} \theta$ where $\theta$ is the maximum angle of deformation. This results in a substantial increase in computational cost for larger $\theta$. Hence in the modified deforming cell method developed in this study, the maximum angle of deformation before realignment was reduced from $45^{\circ}$ to $26.6^{\circ}$. The new angle corresponds to the minimum angle at which the cell can be realigned without effecting the message passing pattern. The overhead in number of interactions considered for the new method is about $40 \%$ compared to $180 \%$ for the previous method.

It is important to note that there is a message passing overhead for the realignment process which is required because the processors would no longer represent the same sub domains in the cell before and after realignment. However, this overhead is very small compared to the total simulation time because it is not attempted at every time step of the simulation.

\section{Results}

The results presented in this paper were obtained using the WCA fluid system whose interatomic potential is the Lennard-Jones potential truncated at the minimum energy and shifted so that both potential and force go to zero at the cutoff. All the simulations were conducted at the LJ triple point $\left(T^{*}=0.722\right.$ and $\left.\rho^{*}=0.8442\right)$. A reduced time step of 0.003 was used. Intel Paragon XP/S 35 and XP/S 150 were used for the simulations.

The scaling results of the NEMD code developed in this study are presented as a $\log -\log$ plot of time per time step against the number of processors for different sizes in figure 1. For a code that scales linearly with number of particles and has minimum communication costs, the curves should be linear with a slope of -1 for a constant system size. We can see that the code scales linearly with better performance for larger system sizes. This improvement in performance is expected due to the fact that for larger systems, the size of the system or subdomain on each processor is considerably large compared to its skin (particles in the link cells at the subdomain boundaries which account for all the message passing).

Figure 2 shows the performance of the new method as compared to that of Hansen and Evans in terms of time taken per time step against number of particles. The simulations were run on 125 processors with same code but using corresponding deformation angles. Also included are the EMD results obtained under same conditions. The EMD results represent the limiting case of zero overhead due to the periodic boundary conditions. We 
can see that the new method with shorter realignment angle performs significantly better than the previous method [3](i.e. the one using $45^{\circ}$ realignment angle). We can also see that the performance of the present method is close to the EMD code, suggesting that the overhead added to the simulation over the equilibrium case by the deforming cell boundary conditions can be significantly reduced by this method.

The new algorithm allowed us to study the rheological properties of the WCA fluid over a wide range of shear rates. Large scale simulations are necessary for such studies in order to obtain good statistical results especially at low shear rates where the signal to noise ratios are unfavorable because of the weak shear fields. Simulations of up to 500,000 particles were conducted on the Paragon XP/S 35 and 150 using up to 343 processors. The viscosity results obtained from these simulations are shown in Figure 3. These results, for the first time, quantitatively show the transition from Newtonian to non-Newtonian behavior. They also show the consistency of the NEMD results in the Newtonian regime with the linear viscosity result obtained using Green-Kubo calculations.

\section{Conclusions}

In this work we have developed an efficient form of the parallel deforming cell NEMD method that significantly reduces the penalty caused by the periodic boundary conditions. This method can be implemented with any NEMD algorithm (either sequential of parallel) which uses linkcell technique to obtain similar improvements in performance. We have also demonstrated the need for large scale parallel NEMD simulations to gain a better understanding of the shear flow properties

\section{Acknowledgements}

The work of HDC has been supported by the Division of Chemical Sciences of the U. S. Department of Energy (DOE) at Oak Ridge National Laboratory (ORNL).This work was sponsored by the Laboratory Directed Research and Development Program of ORNL. The authors acknowledge the use of the Intel Paragon supercomputers in the Center for Computational Sciences at ORNL, funded by the DOE's Mathematical, Information, and Computational Sciences Division. ORNL is managed by Lockheed Martin Energy Research Corp. for the DOE under Contract No. DE-AC05-96OR22464.

\section{References}

[1] Pinches, M.R.S., D.J. Tildesley, and W. Smith, Large scale molecular dynamics on parallel computers using the link-cell algorithm. Mol. Sim., 1991. 6: p. 51. 
[2] Rastogi, S.R. and N.J. Wagner, A parallel algorithm for Lees-3 boundary conditions. Parallel computing, Submitted for publication, 1995.

[3] Hansen, D.P. and D.J. Evans, A parallel algorithm for nonequilibrium mol ecular dynamics simulation of shear flow on distributed memory machines. Mol. Sim., 1994. 13: p. 375.

\section{DISCLAIMER}

This report was prepared as an account of work sponsored by an agency of the United States Government. Neither the United States Government nor any agency thereof, nor any of their employees, makes any warranty, express or implied, or assumes any legal liability or responsibility for the accuracy, completeness, or usefulness of any information, apparatus, product, or process disclosed, or represents that its use would not infringe privately owned rights. Reference herein to any specific commercial product, process, or service by trade name, trademark, manufacturer, or otherwise does not necessarily constitute or imply its endorsement, recommendation, or favoring by the United States Government or any agency thereof. The views and opinions of authors expressed herein do not necessarily state or reflect those of the United States Government or any agency thereof. 


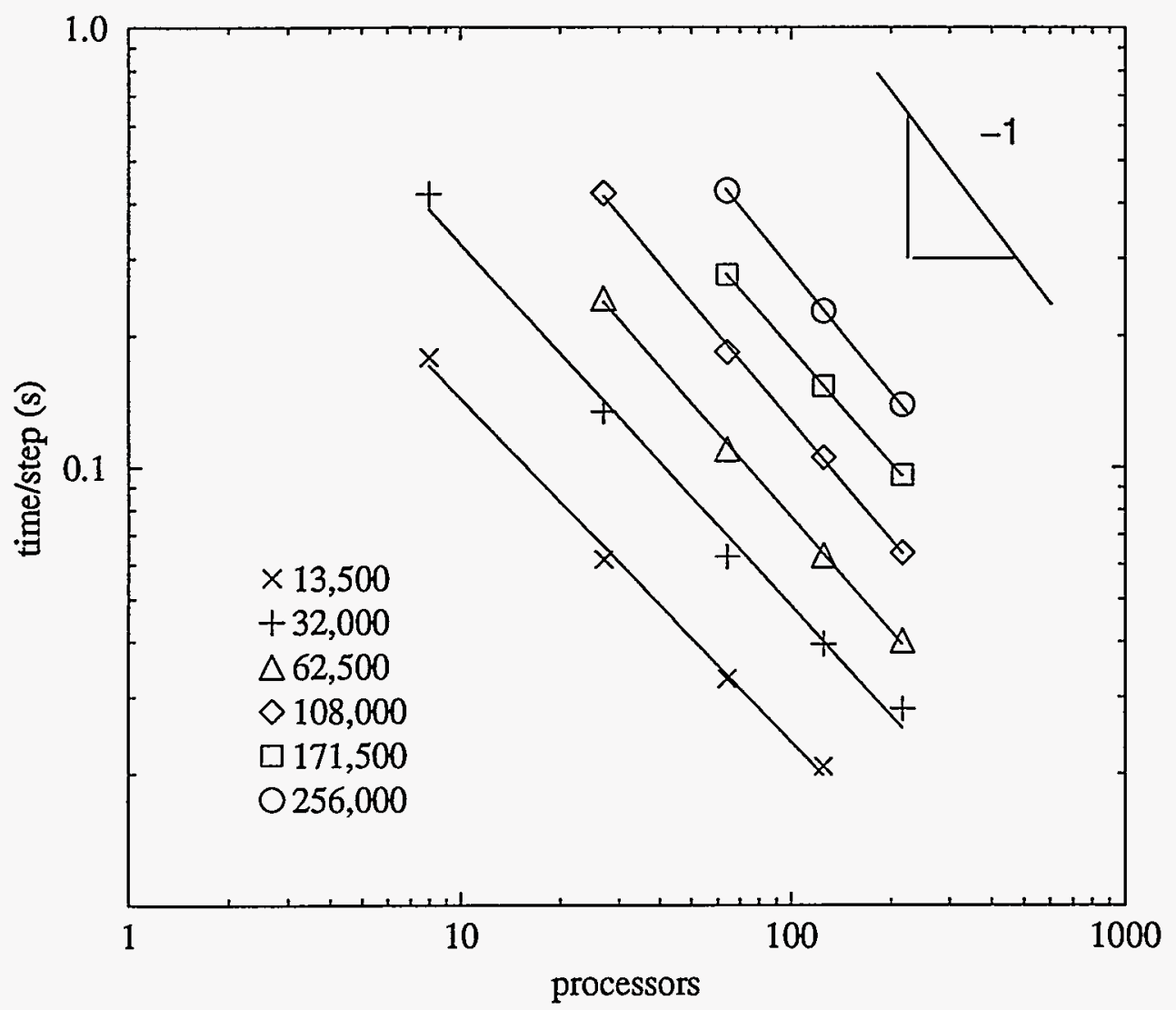

Figure 1: Scaling results for the NEMD code with $26.6^{\circ}$ realignment angle. 


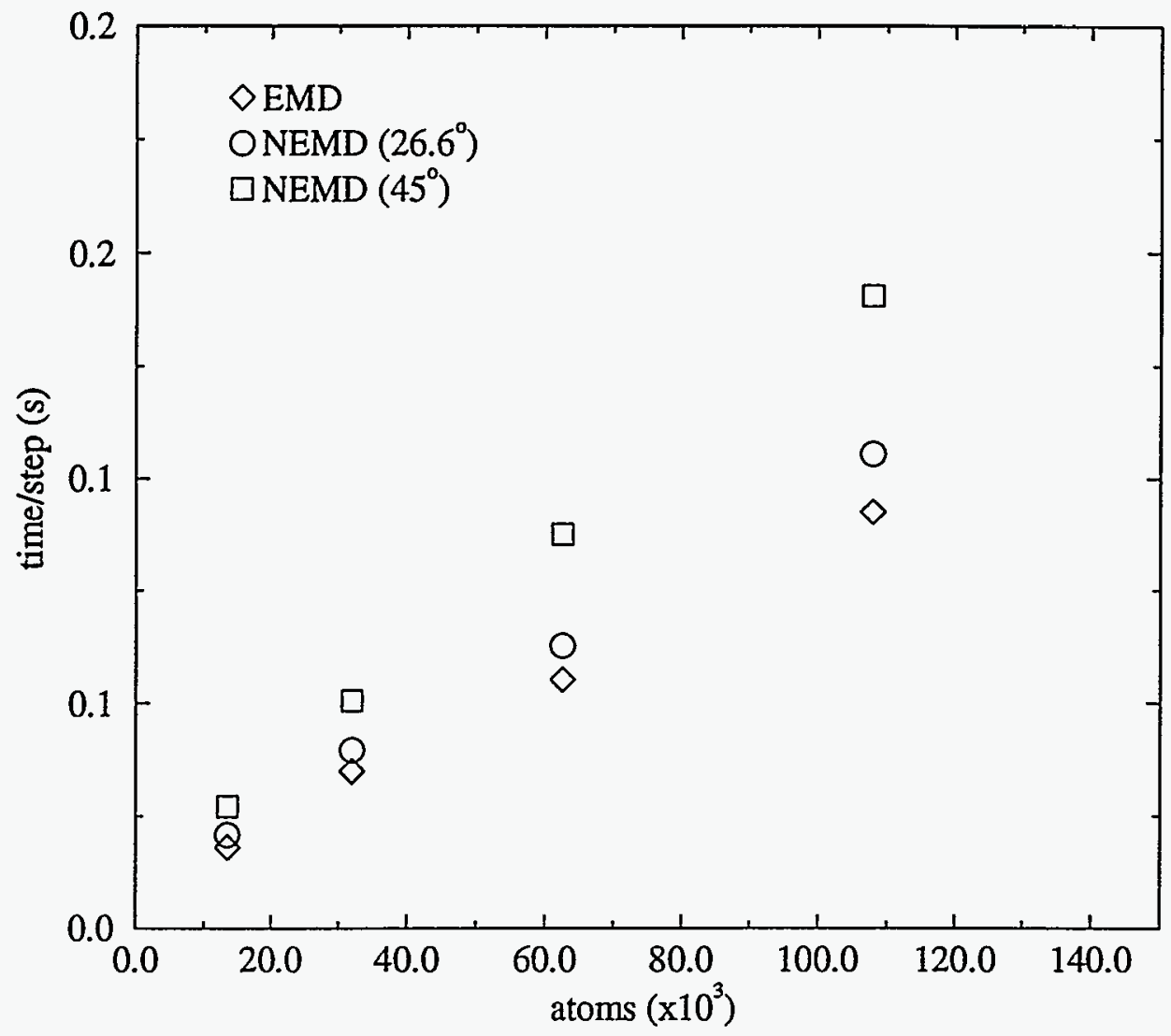

Figure 2: Comparision of the performance of the new method and the Hansen and Evans [3] method. 


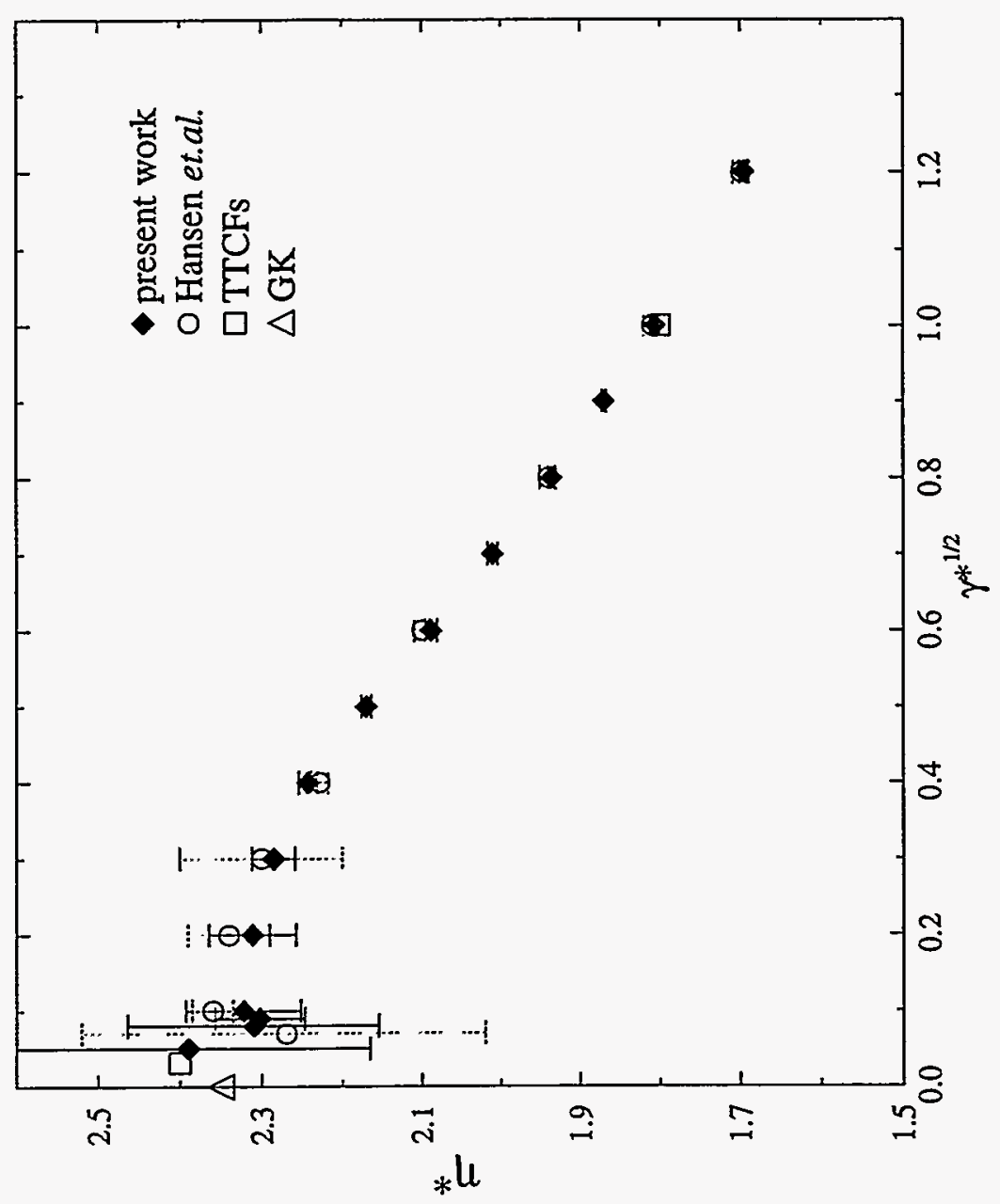

Figure 3: Shear viscosity results for the WCA fluid system. 